Methods All exposed HCWs working in King Abdullah Bin Abdulaziz Specialized Children's Hospital exposed to measles, rubella, mumps, and varicella during 2017 and 2018 were identified through active investigation and passive reporting. A multi- partner intervention was done during December 2017 to reduce the risk and outcome of exposure. Immune status of exposed HCWs as previously documented in the electronic records was evaluated. Those who were not sufficiently immune were given the relevant vaccine (MMR or varicella). Group and individual education was done to increase the awareness of HCWs. Engagement of departmental heads and nurse managers was pursued to encourage compliance. The outcomes of post-exposure management were compared before (2017) and after (2018) starting the intervention.

Results A total of $213 \mathrm{HCWs}$ were exposed to the targeted infectious diseases (97 in 2017 and 116 in 2018). Of 213 HCWs, $41.3 \%$ were exposed to varicella, $41.3 \%$ to mumps, $8.9 \%$ to measles, and $8.5 \%$ to rubella. Compliance with postexposure evaluation improved from $74.5 \%$ in 2017 to $95.0 \%$ in 2018. Although more HCWs were exposed to one of the above diseases in 2018 compared with 2017, the immune status of HCWs significantly increased from $69.4 \%$ in 2017 to $91.7 \%$ in $2018(\mathrm{p}<0.001)$. Cleared HCWs increased from $68.2 \%$ in 2017 to $90.1 \%$ in $2018(\mathrm{p}<0.001)$. Those who were granted sick leave decreased from $2.3 \%$ in 2017 to $0.7 \%$ in $2018(\mathrm{p}=0.573)$.

Conclusion A post-exposure intervention including immunization and awareness was successful at improving immunity and return to work rates, and reducing the need for sick leave. This intervention needs to be continuously implemented, especially in high-risk locations such as emergency departments. This can probably increase the safety of the work environment and reduce related absenteeism.

\section{CONSUMPTION OF RAW MILK IS THE MAIN CAUSE OF BRUCELLOSIS IN THE NATIONAL GUARD POPULATION AT RIYADH: TIME TO CORRECT THE MISCONCEPTION}

Hedaih Alotaibi, Aiman El-Saed Ramadan, Abrar Turkistani. Infection Prevention and Control Department, King Abdulaziz Medical City

\subsection{6/bmjoq-2019-PSF.62}

Background Human brucellosis is one of the most common communicable diseases in Saudi Arabia. It affects a large number of the population and causes high morbidity, mortality, and healthcare cost. People usually get the disease after exposure to infected animals or animal products contaminated with the bacteria. A significant steady increase in the number of brucellosis cases reported to the National Guard Hospital in Riyadh has been noticed during the past 5 years. The number of cases increased from 301 patients in 2013 to 449 patients in 2017.

Methods Serology and microbiology results of new brucellosis diagnoses are reported weekly to the public health section of the Infection and Control Department, National Guard Hospital, Riyadh. In response to the increasing number of cases of brucellosis, patients that were reported in 2017 were contacted by phone to assess the method of transmission and to refer family members with suspected exposure to the infectious disease clinic.

Results A total of 449 patients with newly diagnosed brucellosis have been contacted. Approximately two-thirds of the patients were male $(67.7 \%)$ and the average age was 41.3 \pm 21.7 years. The highest number of reported patients was observed in October $(n=57)$. Almost all patients had Saudi nationality $(99.8 \%)$. For the exposure history, the most frequent exposure was drinking raw milk $(n=390,86.9 \%)$. Approximately $10.9 \%$ of the patients had a history of animal exposure during the past 6 months. Approximately 2.2\% of the patients denied all possible exposures.

Conclusion Drinking raw milk is still the most frequent exposure among patients with brucellosis reported to the National Guard Hospital in 2017. There is an urgent need for a more effective health education campaign that puts into consideration the cultural part of the problem. Multiple partners should be engaged including public health, Ministry of Agriculture, media, and community and religious leaders.

\section{PREVALENCE AND RISK FACTORS OF GESTATIONAL DIABETES MELLITUS AMONG PREGNANT PATIENTS ATTENDING NATIONAL GUARD PRIMARY HEALTHCARE CENTERS IN JEDDAH CITY}

Mutaz Fouad Nabrawi, Razaz Mohammed Wali, Mutaz Fouad Nabrawi, Saleem Abdullah Alsaedi, Abdullah Abdulaziz Altalhi, Abdulrahman Abdullah Aldainy. College of Medicine, King Saud bin Abdulaziz University for Health Sciences

\subsection{6/bmjoq-2019-PSF.63}

Background Gestational diabetes mellitus (GDM) is defined as glucose intolerance with onset or first recognition during pregnancy, and is usually screened for at 24-28 weeks of gestation. Risk factors that have been identified include persistent glucosuria, history of macrosomic fetus, obesity, age older than 25 years, congenital malformations, and strong family history of type 2 diabetes, which is a common illness in our country. In our study, we estimated GDM prevalence and evaluated its risk factors among female patients attending National Guard Primary Healthcare Centers in Jeddah city in 2017.

Methods This was an observational cross-sectional study. Our sample size was calculated to be 347 . We included all pregnant females, aged $15-45$ years, who attended National Guard Primary Healthcare Centers in Jeddah, and had been following up there during the study period. We collected data from antenatal visit notes, and labor and delivery sheets using the electronic file system BestCare. Screening for GDM at 24-28 gestational weeks was done using the American Diabetes Association (ADA) two-step approach, starting with 1 hour $50 \mathrm{~g}$ glucose challenge test, followed by 3 -hour $100 \mathrm{~g}$ glucose tolerance test. We used SPSS 24.0 to analyze data.

Results The prevalence of GDM among our population was calculated to be $19.6 \%$. Glucose challenge test was abnormal in $36.6 \%(n=127)$ of the sample, and $6.9 \%(n=24)$ had diagnostic value. Glucose tolerance test was abnormal in $18.7 \%$ $(n=65)$ of the sample, and 15\% $(n=52)$ had diagnostic value. Several factors were significantly associated with GDM including age $(p<0.001)$, height $(p=0.028)$, and body-mass index (BMI; $\mathrm{p}=0.045)$.

Conclusion Prevalence of GDM is considered high among our population. Dietary habits and high BMI play an important role in the increasing amount of GDM cases. It is important to prevent GDM to minimize risks for both the mother and fetus. 